\title{
¿Cómo medir los flujos migratorios?
}

\author{
Úrsula Faura Martínez \\ Juan Gómez García
}

Universidad de Murcia. Departamento de Métodos Cuantitativos para la Economía y Empresa Campus de Espinardo. 30100 Murcia. Spain

faura@um.es

\section{Resumen}

El fenómeno migratorio, como determinante de la distribución poblacional, tiene gran importancia y, por ello, es muy elevado el número de trabajos dedicados al análisis del mismo. Sin embargo, no existe homogeneidad conceptual ni metodológica en su tratamiento. La no disposición de datos directos y, por tanto, la necesidad de estimarlos a partir de diferentes fuentes añade más dificultad a la interpretación y comparación de los resultados de los diferentes trabajos. El objetivo de este articulo es revisar los conceptos y métodos que se utilizan en el análisis del tema, facilitando la comparación de los resultados de los diferentes trabajos.

Palabras clave: migración, tasas migratorias, índice de efectividad.

\section{Abstract. How to measure migratory flows?}

The migratory phenomenon, like determinants of the population distribution, has great importance and, for that reason, very is raised the number of jobs dedicated to the analysis of the same one. Nevertheless, conceptual nor methodology homogeneity in its processing does not exist. The nondisposition of direct data and, therefore, the necessity to consider them from different sources add to more difficulty to the interpretation and comparison of the results of the different works. The objective of this articulate is to review the concepts and methods that are used in the analysis of the subject, facilitating the comparison of the results of the different works.

Key words: migration, rates, index of effectiveness.

\section{Sumario}

1. Introducción

2. Definición de migración

3. Medición de los flujos migratorios
4. Aplicación al estudio de las migraciones en España durante el periodo 1985-1995

Bibliografía 


\section{Introducción}

La migración es un fenómeno demográfico muy diversificado que no posee la claridad conceptual que tienen los otros procesos demográficos como nacimiento y muerte, de ahí la complejidad de su estudio y la variedad de trabajos que tratan sobre el tema. Se diferencia de los otros dos componentes básicos del cambio de población (natalidad y mortalidad) ${ }^{1}$ en los siguientes aspectos:

1. No es un proceso biológico.

2. Los movimientos implican siempre salida de una región y entrada a otra, y la natalidad y mortalidad sólo se refieren a una de ellas.

3. Natalidad y mortalidad son sucesos universales, es decir, si el fin de la sociedad humana es sobrevivir, ello requiere reproducción y algún control sobre la muerte, mientras que la migración es un aspecto opcional de la acción humana.

4. La decisión de migrar, no significa sólo salida de una región y entrada a otra, sino, además, un aspecto relacionado con el no-movimiento, pues no existe sólo la opción de moverse, sino también la de no moverse.

Hay dos aspectos fundamentales relacionados con el proceso migratorio. El primero referente a la explicación de un modelo particular de migración (determinantes) y el segundo relacionado con la predicción. No solo hay que conocer las causas y el proceso de migración, sino también el flujo de migración actual y poder predecir futuros movimientos de población. Los estudios de la migración se han centrado fundamentalmente en los determinantes frente a las consecuencias de la migración. Pero es necesario partir de un concepto claro, que permita poder comparar distintos trabajos. Este artículo pretende aclarar las bases conceptuales y metodológicas más generales que se utilizan en los estudios sobre migración. Por ello, comenzaremos definiendo lo que es una migración en el epígrafe 2, y cómo se miden los flujos migratorios a partir de los datos disponibles en el epígrafe 3. Además, estudiamos los flujos en España por comunidades autónomas durante el periodo 1986-1995, desde un punto de vista descriptivo, analizando las distintas medidas sobre migración, viendo las ventajas de unas sobre otras y la correlación existente entre ellas.

\section{Definición de migración}

Frente a los fenómenos demográficos de natalidad y mortalidad, la migración plantea diversas cuestiones conceptuales. Mientras los dos primeros son fenómenos biológicos que no presentan ningún problema a la hora de su registro y cómputo, las migraciones son transiciones espaciales, temporales y sociales a la vez, sobre las que no existe un consenso generalizado. Shaw (1975) considera que una definición comprensiva y universal no existe y se lamenta de este problema en su trabajo.

1. Goldscheider (1971), p. 48-51. 
Tabla 1. Algunas definiciones de migración.

\section{Definición}

Fuente

Una persona es un migrante si expresa su intención

de moverse próximamente.

Rossi (1955)

Una migración es un cambio de residencia que envuelve un cambio completo y un reajuste de las afiliaciones comunitarias del individuo.

Una persona es un migrante si abandona su región de nacimiento.

Una migración es un cambio de residencia, independientemente de la distancia.

Un migrante es una persona que cambia de trabajo y en dicho proceso cruza los límites de una región a otra. Schwartz (1976) La migración es un cambio permanente en el lugar de residencia cruzando bordes específicos administrativos o políticos.

Bogue (1959)

Musgrove (1963)

Lee (1966) aninistrativos o politicos.

Nurun y Krishnan (1993)

Por otro lado, normalmente los datos migratorios no son susceptibles de medición directa y deben ser inferidos a partir de alguna fuente. Esto determina que muchas veces la definición esté condicionada por el estudio realizado a partir de las fuentes estadísticas y dependerá de lo que se pretenda estudiar y de la información disponible.

La mayoría de las definiciones envuelven un cambio de la residencia usual de una persona o familia. Este cambio puede realizarse a una distancia muy pequeña (dentro de la misma urbanización) o a una distancia muy grande (entre países). Hay situaciones en las que es difícil determinar qué constituye una migración, cómo pueden ser los estudiantes universitarios que van y vienen entre la vivienda familiar y la de la región de estudio, o aquéllos que poseen varias viviendas y pueden establecerse en una u otra cuando quieran. Por ello, es necesario incluir en la definición de migración una componente temporal, para distinguir entre movimientos temporales y permanentes.

En la tabla 1 hemos reflejado algunas definiciones, como la de Rossi (1955), que define a los migrantes o migrantes potenciales en términos de sus intenciones migratorias ${ }^{2}$. Lee (1966) entiende por migraciones todos los movimientos que conlleven un cambio de residencia permanente o semipermanente, sin hacer caso a la distancia, y referidos a movimientos forzados y a voluntarios ${ }^{3}$. De esta forma, contabiliza como migración un cambio de vivienda dentro de la misma unidad administrativa (como, por ejemplo, el municipio).

Schwartz (1976) está interesado en la relación existente entre migración y mercado laboral, definiendo el migrante como alguien que cambia de traba-

2. Otros trabajos que consideran al migrante en términos de sus probabilidades de migración son Wolpert (1965), Brown y Moore (1968), entre otros.

3. Una definición en términos parecidos puede encontrarse en Thomlinson (1962). 
jo (o está buscando uno) y en dicho proceso cruza los límites de una región a otra. Dada esta definición, el conjunto de migrantes es un subconjunto de los que buscan trabajo.

Según Beaman y Dárcy (1980), el problema que existe con el concepto de migración se puede solucionar en parte, si se considera la migración desde una perspectiva multivariante, es decir, distinguiendo varios aspectos dentro de ella, como MCginnis (1968) y MCfarland (1970), que estudian la migración tomando como referencia el tiempo que ha permanecido en la región, junto con otros factores como la edad. La ONU recomienda distinguir los migrantes que se establecen, los de largo período (duración de la estancia superior a un año) y los de corto período (estancia inferior a un año).

Para Puyol (1990) una migración es un conjunto de movimientos que tiene por objetivo trasladar la residencia de las personas interesadas de un lugar de origen a otro de destino. Una migración supone siempre el desplazamiento de un grupo importante de personas y no tiene porque ser definitiva (desplazamientos continuos, estacionales o diarios).

En Nurun y Krishnan (1993) la migración es definida como un cambio permanente del lugar de residencia cruzando bordes específicos administrativos o políticos. Además, distinguen, para áreas acotadas, entre movimiento y migración; al igual que entre migración interna e internacional. Un movimiento es un cambio de residencia dentro de los mismos límites políticos o administrativos, y una migración es un cambio de residencia y también un cambio sobre los límites políticos o administrativos. Migración interna es un cambio del lugar de residencia de un límite administrativo a otro dentro del mismo país, y migración internacional es una migración sobre el límite nacional.

En Shen (1994) se distingue entre transición y movimiento. Por transiciones entiende el número de migrantes que se mueven dentro de un período fijo o variable, de forma que cada migrante es contabilizado tan sólo una vez. Para medir la migración toma el número de movimientos realizados por los migrantes en un período de tiempo. Cada movimiento es contado una sola vez, pero un migrante puede realizar múltiples movimientos en un período y ser contado varias veces.

Vinuesa et al. (1994) definen la migración como un cambio de residencia desde una unidad espacial de rango administrativo hacia otra, en un intervalo de tiempo fijado a priori. Podemos encontrar otras definiciones en Puyol (1990), Romero González y Albertos Puebla (1993) y Zax (1994).

\section{Medición de los flujos migratorios}

La literatura sobre migración tampoco presenta una posición común respecto a las medidas del fenómeno migratorio. La medición puede referirse a la migración bruta, es decir, a las salidas del territorio por un lado y a las entradas por otro, o a la migración neta, saldo de las entradas y salidas. Estos datos pueden obtenerse mediante observación directa del movimiento, o bien de una manera indirecta. La migración bruta generalmente se calcula a partir de las observaciones direc- 
tas. La migración neta, que por definición no es un dato de observación, se obtiene muchas veces por métodos de estimación indirecta que no implican el conocimiento de los flujos brutos. Hay, además, divergencias en las definiciones de tasa de migración, en la construcción de tablas de vida que incluyen flujos migratorios e incluso diferencias entre si considerar migraciones o migrantes. A continuación, exponemos diferentes procedimientos para realizar estos análisis.

\subsection{Método exacto}

El método ideal mediante el cual se podría conocer la migración neta en un período dado, requeriría conocer los datos de la población inicial y final, los nacimientos y muertes en la región, la migración bruta y también datos que indiquen la emigración y la inmigración entre el crecimiento natural o el crecimiento natural entre los migrantes. Pero no existe ninguna estadística o estadísticas a partir de las cuales se puedan conocer todos estos datos. Sería necesario un registro continuo diseñado para contabilizar el crecimiento natural y la migración en el momento en el que ocurren. Si se dispusiera de tales datos, se podría conocer con exactitud el número total de nacimientos, muertes y migraciones efectuadas en el período y así el crecimiento de la población.

\subsection{Métodos de estimación}

Como no se dispone de la información necesaria para obtener de forma exacta los datos referentes a la migración neta, el cálculo de la misma estará en función de la información de que se disponga. Los tres métodos más usados son ${ }^{4}$ : la estimación a partir de la información sobre el lugar de nacimiento, el método del balance migratorio y el método de la población esperada.

\subsubsection{A partir de la información sobre el lugar de nacimiento}

Una medición de la migración se puede obtener utilizando los censos de población desde el momento que se conoce la localización de los individuos en una fecha anterior al momento de la observación, como lugar de nacimiento, lugar de residencia en el momento del censo precedente (o en un momento dado cualquiera anterior al censo) y de la duración de la residencia.

Si no hay preguntas directas sobre migración en el censo, se pueden obtener de una manera indirecta estimaciones de la migración neta, a partir de la información sobre el lugar de nacimiento y el lugar de residencia. Esta forma tiene la ventaja de que utiliza sólo un censo, pero con este procedimiento se calculan migrantes y no migraciones; se ignoran las migraciones

4. Los distintos métodos no conducen necesariamente a los mismos resultados. El método de balance migratorio y el de la población esperada ofrecen resultados comparables entre sí, cosa que no sucede con la migración obtenida a partir del censo. Hamilton $(1966,1967)$ realiza una comparación pormenorizada de los distintos métodos. 
múltiples, los retornos a la población de origen y la incidencia de la mortalidad. Cuando se dispone, en un censo, a la vez del lugar de residencia anterior y de la duración de estancia en la residencia actual, es posible calcular la última migración.

Si se compara el lugar de residencia entre dos censos consecutivos, se puede estimar la migración neta a partir de la siguiente expresión:

$$
\text { Migración neta }=[I(t+n)-I(t)]-[E(t)+E(t+n)]
$$

con $I(t)$ y $E(t)$ el número de inmigrantes y emigrantes supervivientes en el período $t$, respectivamente. Si se tiene en cuenta la mortalidad, la fórmula sería:

$$
\text { Migración neta }=[I(t+n)-E(t+n)]-[S(i) I(t)-S(0) E(t)]
$$

siendo, $S(i)$ y $S(0)$ los coeficientes de supervivencia intercensal que dan, respectivamente, las proporciones de $I(t)$ y $E(t)$ que sobrevivirán en dicho período. La mayor dificultad de este método radica en la estimación de $S(i)$ y $S(0)$.

\subsubsection{Método del balance migratorio}

Es posible estimar la migración neta intercensal a partir del crecimiento natural de la población entre los dos censos, restándole al cambio poblacional entre dichos censos el crecimiento natural. Este método también se conoce como «método del movimiento natural, estadística vital o de la componente intercensal». La ecuación que se usa tiene la forma de una ecuación de balance:

$$
\text { Migración neta }=[P(t+n)-P(t)]-[N-D]
$$

siendo $P(t)$ y $P(t+n)$ la población total al comienzo y al final del intervalo censal respectivamente, $N$ el número de nacimientos y $D$ el de defunciones en dicho período.

Las ventajas de este método son su simplicidad y cálculo sencillo. Sin embargo, sus resultados estarán en función de la calidad de los datos censales. Al calcularse la migración de forma residual, se tiende a imputar cualquier diferencia al saldo migratorio, es decir, cualquier error porcentual en el estoc de población de magnitud superior al saldo, se traslada íntegramente al cálculo de la migración neta, ya que el crecimiento natural se obtiene a partir de otra fuente. Además, presenta el inconveniente de proporcionar un valor para el conjunto intercensal, sin que sea posible descomponerla en períodos más cortos (el año, por ejemplo). Por otro lado, supone que no hay migración internacional, y si ésta fuese importante se obtendrían resultados de poca fiabilidad 5 .

5. Una explicación muy detallada de los problemas derivados de la aplicación de este método, puede verse en Courgeau (1988), cap. VIII. 


\subsubsection{Método de la población esperada}

Se basa en las probabilidades de supervivencia entre dos censos. Para aplicar este método hace falta conocer la población por edad y sexo entre dos censos sucesivos y un conjunto de tasas de supervivencia. Las tasas de supervivencia se aplican a la población del primer censo, para obtener una estimación del número de personas que se espera sobrevivan en el segundo censo. La diferencia entre la población enumerada en el segundo censo y la población estimada es la migración neta. Se llama también «método del coeficiente de supervivencia» y se calcula como:

$$
N_{1}(x)=P(x+n, t+n)-S \cdot P(x, t)
$$

siendo $N_{1}(x)$ la migración neta para el grupo de edad $x ; P(x, t)$, la población de edad $x$ en el instante $t$ y $S$, el coeficiente de supervivencia.

La proyección puede ser realizada también de manera retrospectiva, a partir de la población observada al final del período:

$$
N_{2}(x)=\frac{P(x+n, t+n)}{S}-P(x, t)
$$

Los dos métodos dan resultados distintos. Se obiene una estimación más realista y fiable mediante la media de los valores obtenidos a través de los dos procedimientos.

El método de la población esperada supone que la población nacional es cerrada, es decir, es insignificante la migración internacional; que las tasas específicas de mortalidad son iguales para cada región y para toda la nación y que la descomposición por grupos de edad y sexo para cada región y para el total nacional es la misma para los dos períodos intercensales. Si no se verifican estas hipótesis, las estimaciones que se obtengan por este método no serán buenas y sería necesario realizar algún tipo de ajuste para mejorar la calidad de las estimaciones.

Pero, en general, la migración neta es una estimación sin ninguna medida de error o precisión de la misma. Por ello sería conveniente dar una medida formal de la incertidumbre sobre el valor estimado y mejorar así las proyecciones que se realizan usando la migración neta. Es importante, además, conocer el nivel de confianza de dicha estimación. En este sentido, Kintner y Swanson (1993) presentan un método para obtener intervalos de confianza alrededor de la migración neta estimada, cuando las estimaciones están realizadas por este método, tomando las tasas de mortalidad como variables aleatorias, aunque ésta no es la única fuente de error en la estimación de la migración neta.

\subsection{Tasas de migración}

Al igual que se obtienen tasas de fecundidad y mortalidad, se calculan tasas de migración para el conjunto de la población, permitiendo distinguir entre emigración, inmigración y migración neta. La literatura sobre migración presenta una posición un tanto ambigua con respecto al cálculo y presentación de 
tasas de migración. El problema ${ }^{6}$ de elegir una tasa de migración adecuada se complica debido a:

1. La variedad de tipos y fuentes de datos sobre migraciones.

2. La variedad de definiciones y formas de estimar las migraciones.

3. La interacción y las relaciones recíprocas entre las migraciones y el crecimiento natural.

4. Las técnicas de cálculo disponibles.

Una tasa de migración $(T M)$ se define como un porcentaje que representa el número de movimientos migratorios realizados $(M)$ sobre el número total de personas $(P)$ durante el intervalo dado $T M=(M / P) \cdot 100$. Si se quiere obtener una tasa de dimensión anual, se debe dividir el número de migraciones por la amplitud del intervalo considerado.

La tasa de emigración $(T E)$ y de inmigración $(T I)$ son tasas migratorias referidas respectivamente a las migraciones de salida y a las de entrada. El problema radica en qué escoger como denominador de las tasas, si la población al comienzo del período, al final del período, la media de ambas, la población de la región de origen, de destino, etc. ${ }^{7}$. La población inicial es la más utilizada, pero presenta algunas debilidades matemáticas y lógicas. La población inicial no representa la verdadera población en riesgo, su uso, si hay una gran inmigración, no sería adecuado, no es totalmente satisfactorio para el caso de la emigración. Y el cálculo de una tasa de inmigración que estableciera la relación del número de inmigrantes de un período sobre la población de origen, es decir, sobre la población del resto del mundo, es de difícil aplicación. El uso de la población final de la región de destino como denominador de la tasa de inmigración es muy pobre y para la tasa de emigración no sería válido en cuanto que no representa la población en riesgo.

Para no tener que utilizar un denominador distinto para cada tasa, se suele usar la media de la población inicial y final de la región de origen. La tasa de emigración viene dada por:

$$
T E=\frac{E}{\frac{1}{2}(P(t)+P(t+n))} \cdot 100
$$

la tasa de inmigración ${ }^{8}$ es:

$$
T I=\frac{I}{\frac{1}{2}(P(t)+P(t+n))} \cdot 100
$$

6. Para un análisis más detallado, se puede consultar Hamilton (1965).

7. Sobre qué criterio utilizar, ver Haenszel (1967) y Vanderkamp (1976).

8. Vinuesa et al. (1994), p. 146, consideran que no es correcta la denominación de «tasa de inmigración» para esta expresión, por no tener en el denominador la población sometida a riesgo, y lo llaman «índice de atracción». 
Otras expresiones para las tasas de emigración e inmigración serían las dadas por Hamilton (1965), tomando como denominador para la tasa de emigración

$$
P(t)+\frac{1}{2}\left[\frac{(N+I-D)}{\frac{1}{2}(P(t)+P(t+n))}\right] P(t)=P(t)+\frac{P(t)}{P(t)+P(t+n)}(N+I-D)
$$

siendo el término entre corchetes la tasa de crecimiento debido al incremento natural y la inmigración. En el caso de inmigración, en el denominador debería aparecer

$$
P(t+n)-\left[\frac{P(t+n)}{P(t)+P(t+n)}\right](N-D-E)
$$

en el que se admite que el límite inferior de la inmigración está representada por la población al final del período menos

$$
\frac{P(t+n)}{P(t)+P(t+n)}
$$

del cambio debido al crecimiento natural y a la emigración. En ambos casos, los denominadores representan una aproximación al número de períodos hombre en riesgo, dado por

$$
Y=\int_{t}^{t+n} P(t) e^{r t} d t=\frac{(P(t)-P(t+n)) \cdot t}{\ln \left(\frac{P(t+n)}{P(t)}\right)}
$$

siendo $r$ la tasa continua de crecimiento anual.

Para una región dada, la tasa de migración neta $(T M N)$, es la diferencia entre la tasa de inmigración y la tasa de emigración. Teniendo en cuenta las definiciones anteriores, con (1) y (2), la tasa de migración neta se calcula como la relación de la migración neta sobre la población media del período?

$$
T M N=\frac{M N}{\frac{1}{2}(P(t)+P(t+n))} \cdot 100
$$

Cuando se trabaja sobre migrantes y no migraciones, se puede calcular la proporción de emigrantes durante el período considerado, definida como la rela-

9. Rogers (1990) considera que ésta es la forma correcta de definir la tasa de migración neta, pues en caso contrario no sería una tasa demográfica correcta, al no medir la frecuencia de ocurrencia de un suceso demográfico en la población en riesgo. 
ción entre el número de emigrantes durante el período considerado y la población inicial que sobrevive al final del período. La proporción de inmigrantes es el cociente entre el número de inmigrantes y la población de la región de destino al final del período. Las mediciones llevadas a cabo sobre migrantes no son aditivas, a diferencia de las realizadas sobre migraciones.

\subsection{Otras medidas}

Se pueden calcular, además de tasas migratorias, una serie de índices relacionados con subgrupos particulares de la población, tales como índices de efectividad, de compensación, de velocidad, de preferencia, de migración diferencial, etc. ${ }^{10}$. Cabe destacar el índice de migración diferencial definido como

$$
d_{m}^{E}=\frac{E_{m} \cdot P}{E \cdot P_{m}}-1
$$

siendo $P_{i}$ y $E_{i}$ la población para distintos grupos $(i=1,2, \ldots, n)$ y el número de emigrantes en cada uno de esos grupos, respectivamente. En la medida en que los individuos del grupo $m$ no emigren a ninguna región, $\left(\left(E_{m} / P_{m}\right)=0\right)$, emigren en la misma proporción que el conjunto de la población, $\left(\left(E_{m} / P_{m}\right)=E / P\right)$, o emigren en su totalidad, $\left(E_{m}=P_{m}\right)$, el índice de migración diferencial es, respectivamente, $-1,0$ y $(P / E)-1$.

El índice de compensación entre dos regiones $i$ y $j$, se define como el cociente entre la migración neta entre $i$ y $j$, y la migración total entre ambas. El índice de efectividad de la región $j$ está dado como la relación entre la migración neta entre $j$ y las demás regiones del sistema (no sólo $i$ ) y la migración total entre $j$ y el resto de las regiones (algunos autores utilizan estos dos conceptos de forma indistinta, nosotros también). Este índice representa el porcentaje de movimiento total que conduce a un cambio de población, expresando el grado por el cual la inmigración y la emigración no se cancelan.

El índice de efectividad de la región $j, C_{j}$, está dado por

$$
C_{j}=100 \frac{N_{j}}{T_{j}}
$$

siendo $M N_{j}=I_{j}-E_{j}$, la diferencia entre la inmigración $\left(I_{j}\right)$ y la emigración $\left(E_{j}\right)$ y $T_{j}$, la suma de ambas, por tanto, es el cociente (en porcentaje) entre la migración neta (inmigración menos emigración) y migración total (inmigración más emigración). El índice será positivo cuando la inmigración sea superior a la emigración, y negativo en caso contrario. Cuando el valor absoluto del índice sea alto, todos se moverán en la misma dirección. Inversamente, un valor bajo

10. Courgeau (1980) realiza un análisis de estas medidas. 
indica que la emigración y la inmigración son prácticamente iguales, se compensan. Es 0 si el flujo migratorio en una dirección coincide con el de la otra, y 1, si sólo hay flujos en una dirección. Schwartz (1976) considera que este índice está normalmente más próximo a la unidad y que refleja el grado de desigualdad salarial entre regiones ${ }^{11}$.

Aunque este índice está usualmente correlacionado con la migración neta, presenta algunas propiedades que lo hacen más atractivo que la tasa de migración neta. La tasa de migración neta se obtiene utilizando la población en el denominador y, por tanto, depende de la historia pasada de cambio de población. De esta forma, algunas regiones pequeñas pueden presentar tasas de migración neta grandes, aunque no sean uno de los destinos más atractivos para la migración. Sin embargo, este índice depende sólo del nivel de movimiento en el período estudiado y, además, nos evitamos el problema de cuál debe de ser el denominador adecuado para la tasa.

Si se conocen los datos migratorios para las distintas regiones que componen el sistema, se puede calcular el índice entre cada par de regiones

$$
C_{i j}=100 \frac{N_{i j}}{T_{i j}}
$$

con $M N_{i j}=X_{j i}-X_{i j}$, la diferencia entre los que llegan a la región $i$ procedentes de la región $j\left(X_{j i}\right)$ y los que se dirigen de $i$ a $j\left(X_{i j}\right)$, y $T_{i j}$, la suma de ambas. Puede definirse un indicador de la eficiencia global, dado por

$$
C=100 \frac{\sum_{j}\left|M N_{j}\right|}{\sum_{j} T_{j}}
$$

siendo $\left|M N_{j}\right|$ el valor absoluto de la $M N$ de la región $j$.

\section{Aplicación al estudio de las migraciones en España durante el periodo 1985-1995}

En España existen principalmente cuatro fuentes de información estadística ${ }^{12}$ sobre migraciones y migrantes: el censo, el padrón municipal de habitantes, la estadística de variaciones residenciales (EVR) y la encuesta de migraciones (EM). El censo y el padrón definen a los emigrantes como aquellas personas de

11. Cuanto mayor sea la diferencia salarial, mayor será el flujo de migrantes hacia la región económicamente superior. Si las dos regiones son económicamente idénticas, el flujo esperado en las dos direcciones se compensará y la migración neta será cero (Schwartz (1976), p. 714).

12. Para estudiar las ventajas e inconvenientes de los distintos métodos se puede consultar Vinuesa et al. (1994) y Ródenas Calatayud (1997). 
diez o más años que en el período intercensal o en otro período fijo, cambiaron su municipio de residencia dentro de la misma región (emigrantes intraregionales) o entre regiones (emigrantes interregionales). Esto permite determinar migrantes y no migraciones, no tiene en cuenta las migraciones múltiples. A partir del padrón de 1986 se formula una pregunta distinta, referida al lugar de la última residencia (incluye a toda la población). La realización del censo y el padrón cada diez y cinco años, respectivamente, unida a la falta de memoria histórica de los entrevistados, hace que éstas no sean buenas fuentes de información estadística.

La EVR, publicada desde 1961, se elabora a partir de los datos individuales acerca de la población migrante que los ayuntamientos remiten al INE. Recogen altas y bajas por cambio de municipio de residencia. Se publica habitualmente en el Anuario Estadístico y, desde 1985, en la serie Migraciones. La ventaja de esta EVR es que ofrece información anual y, por tanto, evita el problema estadístico de la migración crónica, el cual puede ocurrir con los datos del censo. Sin embargo, los datos del censo son más fiables que los de EVR, pues éstos están basados en la declaración individual de cambio de residencia, y muchas personas no registran su cambio de residencia, lo que subestima los datos ofrecidos por esta estadística. Pero estas desventajas son cada vez menos importantes, si tenemos en cuenta que el INE está mejorando la calidad y cobertura de la EVR aplicando métodos de control y recogida de datos cada vez más avanzados. A esto hay que unir la necesidad creciente de tener que presentar el certificado de empadronamiento para poder acceder a un colegio, para obtener empleo público, etc., lo que obliga a realizar la declaración de cambio de residencia.

La encuesta de flujos migratorios, vinculada a la EPA informa, desde su implantación en el segundo semestre de 1987, sobre el flujo de migrantes, tomando como referencia temporal el año transcurrido entre el segundo trimestre del año en curso y el mismo trimestre del año anterior. Esta encuesta sustituye a la anterior encuesta de migraciones interiores, en la que la referencia temporal era distinta, así como la determinación del migrante que implicaba su cuestionario.

Para esta aplicación vamos a utilizar la EVR y, por tanto, definimos «migración interior» como los desplazamientos de individuos entre regiones del territorio español y que se plantean con una duración lo suficientemente amplia como para inducir a las personas a realizar una cambio en el registro de residencia, es decir, con carácter permanente. Cuando la región de procedencia, o la de destino, pertenezca a un país extranjero, hablaremos de «migración exterior».

Ahora bien, los datos ofrecidos por la EVR para los años en los que se ha realizado un censo o padrón están infravalorados, pues durante los meses en los que se efectúa el censo o padrón, los nuevos residentes son dados de alta directamente en el padrón municipal de su nueva residencia y, a la vez, de baja en el municipio de origen, con lo que no quedan registrados en las cifras mensuales de variaciones municipales, de forma que el número de migrantes anual ofrecido por la EVR es más bajo. Esto está reflejado en la tabla 2, en la que 
Tabla 2. Flujos migratorios durante el periodo 1985-1995.

\begin{tabular}{lcc}
\hline Año & No de desplazamientos totales & No de desplazamientos interautonómicos \\
\hline 1985 & 439.305 & 158.028 \\
1986 & 246.953 & 96.806 \\
1987 & 415.700 & 173.401 \\
1988 & 584.950 & 219.998 \\
1989 & 658.129 & 249.828 \\
1990 & 681.488 & 248.448 \\
1991 & 415.700 & 147.882 \\
1992 & 611.026 & 202.852 \\
1993 & 690.059 & 212.725 \\
1994 & 752.089 & 230.334 \\
1995 & 802.925 & 241.279 \\
\hline
\end{tabular}

Fuente: elaboración propia.

se estudia el número de desplazamientos anuales producidos durante el período 1985-1995. Se puede observar el brusco cambio de tendencia de los flujos migratorios en los años 1986 y 1991, correspondientes a un censo y un padrón, respectivamente. Es necesario ajustar los datos para estos años, realizando una interpolación con el año anterior y posterior ${ }^{13}$. Si no tenemos en cuenta las migraciones ocurridas dentro de la misma comunidad autónoma, se observan resultados similares, segunda columna de la tabla 2.

Vamos a comprobar a continuación que la parte más importante para estudiar los cambios en la población es la migración interior. El crecimiento de la población durante el periodo 1986-1995 es debido tanto al crecimiento natural como a las migraciones. La variación de la población durante dicho periodo viene reflejada en el gráfico 1 . Cabe destacar que en los años donde se ha producido un censo o un padrón ${ }^{14}$, el crecimiento de la población es negativo, mientras que en el resto de los años, el crecimiento presenta leves bajadas o subidas.

Pero, ¿cuál es la causa fundamental de estos cambios?, ¿la componente del crecimiento natural o la migratoria? Además, puede ocurrir que no todas las comunidades autónomas contribuyan a estos cambios por la misma causa. Cabré et al. (1988) afirman que los movimientos migratorios son el principal factor diferenciador de la población en el territorio, por su efecto directo sobre el volumen y la estructura de la población, y por su efecto indirecto sobre el crecimiento natural. En esta misma línea encontramos el trabajo de Leguina Herrán y Fernández Cordón (1991), quienes consideran que las migraciones interiores constituyen la variable clave (superior al crecimiento vegetativo) que explica las diferencias de dinamismo demográfico de una región a otra. Para

13. Este método ha sido utilizado por Bentolila y Dolado (1990) y Ródenas Calatayud (1997).

14. Los datos de población referentes a periodos no censales o padronales no siempre son aceptados como válidos, pues hasta hace relativamente poco tiempo no existía un control riguroso sobre las altas y bajas en los registros municipales. 


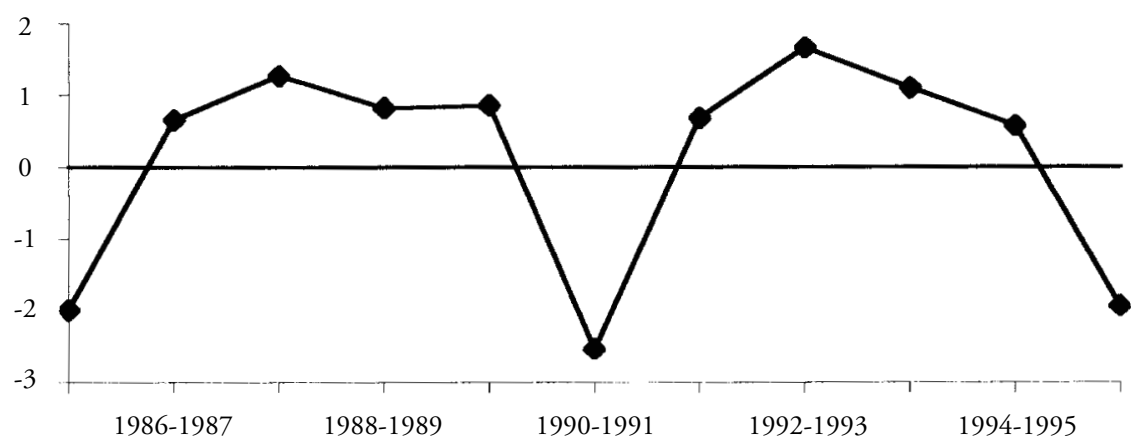

Gráfico 1. Evolución de la población de España (en porcentaje).

comprobarlo, vamos a utilizar un procedimiento residual, que nos indica que la variación conjunta del crecimiento natural del sistema coincide entre comunidades autónomas.

En primer lugar calculamos la tasa de crecimiento total de cada comunidad autónoma, y la tasa de crecimiento de la migración interior, dadas respectivamente por

$$
g_{i}=\frac{1}{n_{i}} \cdot \frac{d n_{i}}{d t} \quad m_{i}=\frac{1}{n_{i}} \cdot \sum_{j \neq i}\left(W_{j i}-W_{i j}\right)
$$

siendo $n_{i}$ la población de la comunidad autónoma $i$ y $W_{i j}$ el flujo migratorio desde la comunidad autónoma $i$ hasta la $j$. Definimos el crecimiento residual (debido al crecimiento natural y a las migraciones externas) mediante $\rho_{i}=g_{i}-m_{i}$. La tasa de crecimiento total de España, vendrá dada por

$g=\sum_{i} \frac{n_{i}}{N} g_{i}=\frac{1}{N} \sum_{i} \frac{d n_{i}}{d t}$, con $N=\sum_{i=1} n_{i}$, siendo el índice de crecimiento residual $\hat{\rho}_{i}=\frac{g_{i}-m_{i}}{g}$. Mediante la varianza de dicho índice (la media vale 1)

$$
\sigma_{\hat{\rho}}^{2}=\frac{1}{L} \sum_{i}\left(\hat{\rho}_{i}-1\right)^{2} \frac{n_{i}}{N}
$$

podemos saber si hay diferencias entre las comunidades autónomas.

En el gráfico 2 se observa que están todas las varianzas alrededor de 0,055, salvo para los años censales 1991 y 1995, en los que aumenta un par de milésimas. Por tanto, el crecimiento natural y la migración externa no son factores diferenciadores entre comunidades autónomas. 
Tabla 3. Migraciones anuales.

\begin{tabular}{lcccrc}
\hline Periodo & $\begin{array}{c}\text { Migraciones } \\
\text { totales }\end{array}$ & \multicolumn{2}{c}{$\begin{array}{c}\text { Migraciones } \\
\text { intrarregionales }\end{array}$} & \multicolumn{2}{c}{$\begin{array}{c}\text { Migraciones } \\
\text { interregionales }\end{array}$} \\
\hline $1986-1987$ & 454.198 & 288.553 & $(63,53)$ & 165.645 & $(36,47)$ \\
$1987-1988$ & 469.240 & 295.839 & $(63,05)$ & 173.401 & $(36,95)$ \\
$1988-1989$ & 584.950 & 364.952 & $(62,39)$ & 219.998 & $(37,61)$ \\
$1989-1990$ & 658.129 & 408.301 & $(62,04)$ & 249.828 & $(37,96)$ \\
$1990-1991$ & 681.488 & 433.040 & $(63,54)$ & 248.448 & $(36,46)$ \\
$1991-1992$ & 646.187 & 420.605 & $(65,09)$ & 225.582 & $(34,91)$ \\
$1992-1993$ & 611.026 & 408.174 & $(66,80)$ & 202.852 & $(33,20)$ \\
$1993-1994$ & 690.059 & 477.334 & $(69,17)$ & 212.725 & $(30,83)$ \\
$1994-1995$ & 752.089 & 521.755 & $(69,37)$ & 230.334 & $(30,63)$ \\
$1995-1996$ & 802.925 & 561.646 & $(69,95)$ & 241.279 & $(30,05)$ \\
$\mathbf{1 9 8 6 - 1 9 9 5}$ & $\mathbf{6 . 3 5 0 . 2 9 1}$ & $\mathbf{4 . 1 8 0 . 1 9 9}$ & $(\mathbf{6 5 , 8 3 )}$ & $\mathbf{2 . 1 7 0 . 0 9 2}$ & $(34,17)$ \\
\hline
\end{tabular}

Entre paréntesis indicamos el porcentaje de migración.

Fuente: elaboración propia.

Demostrado que el principal factor diferenciador en la variación de la población es debido a las migraciones interiores, vamos a estudiar desde un punto de vista descriptivo la evolución de los flujos migratorios, si hay regiones que ganan población de forma sistemática, es decir, atraen más inmigrantes o si, por el contrario, no son atractivas y pierden población; si las regiones con gran inmigración tienen también bastante emigración o si existe alguna relación entre la migración y ciertas variables económicas, etc.

Durante el periodo 1986-1995, hay 6.350.291 movimientos migratorios (tabla 3), de los cuales 4.180 .199 se realizan dentro de la misma comunidad autónoma, es decir, casi el 66\% de los cambios de residencia son intrarregionales. Por años, a partir de 1991 aumenta el número de migraciones dentro de las comunidades autónomas, indicando que las migraciones se producen a

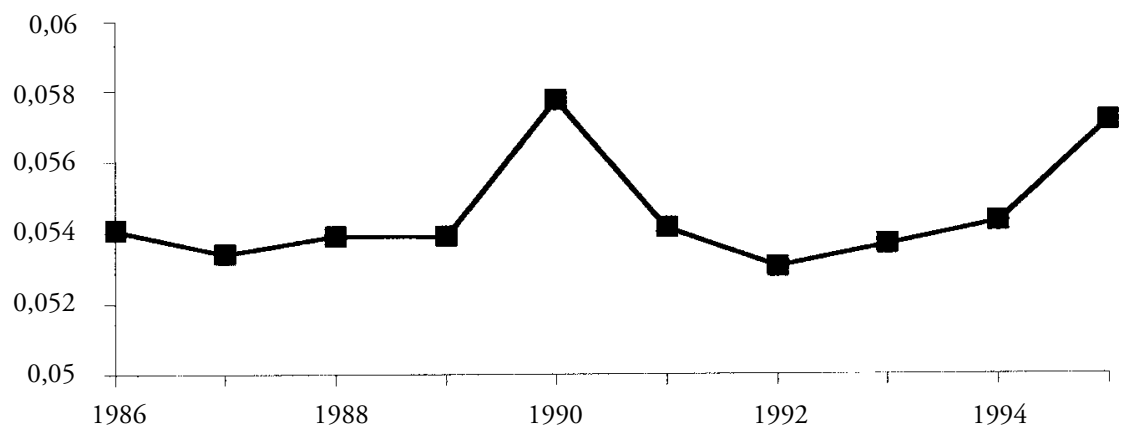

Gráfico 2. Varianza espacial del índice de crecimiento residual. 

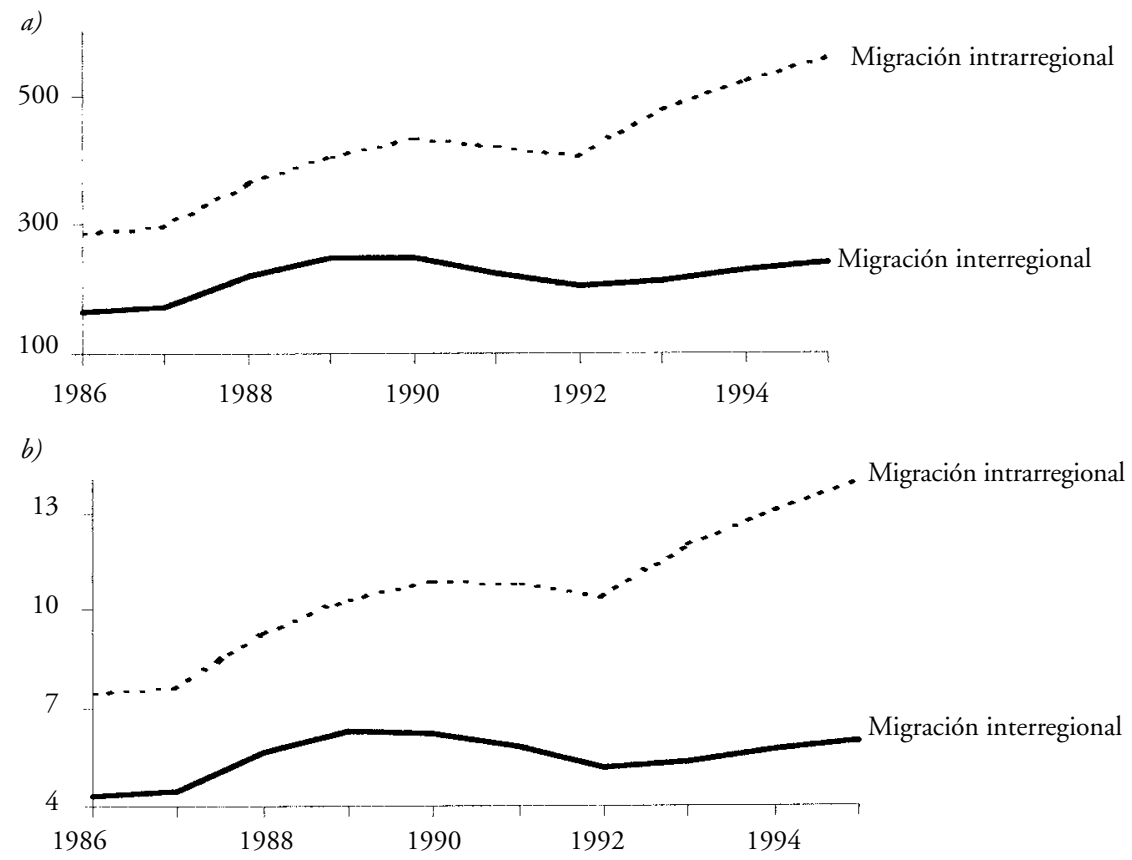

Gráfico 3. a) Volumen total de migración intrarregional y migración interregional en miles, y b) tasa de migración intrarregional y migración interregional en tanto por mil.

Tabla 4. Migración neta por comunidades autónomas.

\begin{tabular}{lrrrrrrrrrr}
\hline & 1986 & 1987 & 1988 & 1989 & 1990 & 1991 & 1992 & 1993 & 1994 & 1995 \\
\hline And. & 1.824 & -800 & -2.709 & -1.327 & 1.668 & 2.249 & 2.830 & 4.648 & 2.591 & -2.795 \\
Arg. & -38 & -309 & 146 & 249 & 567 & 568 & 569 & 493 & 424 & 271 \\
Ast. & -670 & -746 & -1.289 & -826 & -542 & -337 & -128 & -185 & -1.182 & -1.025 \\
Bal. & 1.433 & 5.340 & 9.345 & 7.166 & 1.236 & 921 & 610 & -369 & 2.062 & 4.744 \\
Can. & 5.734 & 8.710 & 9.915 & 6.051 & -1.614 & 530 & 2.672 & 2.836 & 5.214 & 6.824 \\
Cant. & -166 & -253 & -262 & 149 & 58 & 91 & 123 & 505 & 878 & 767 \\
C.L. & -3.341 & -5.424 & -6.708 & -9.248 & -8.653 & -6.729 & -4.804 & -3.140 & -1.205 & -3.062 \\
C.M. & -1.885 & -4.263 & -4.984 & -5.368 & -1.709 & 703 & 3.113 & 3.851 & 5.347 & 5.367 \\
Cat. & -5.448 & -1.714 & 1.321 & 2.142 & 2.169 & -1.158 & -4.486 & -6.210 & -7.259 & -3.549 \\
C.V. & 3.416 & 2.660 & 5.801 & 8.378 & 7.551 & 5.478 & 3.404 & 4.433 & 5.597 & 7.395 \\
Ext. & -476 & -1.610 & -5.267 & -6.391 & -4.514 & -2.487 & -464 & 977 & 1.363 & -464 \\
Gal. & -1.313 & -2.644 & -3.826 & -2.858 & -1.195 & -277 & 642 & 1.146 & 753 & -375 \\
Mad. & 4.742 & 7.690 & 7.671 & 8.702 & 8.948 & 4.103 & -746 & -6.439 & $-11.346-10.713$ \\
Mur. & 1.960 & 1.277 & 886 & 2.152 & 1.160 & 1.493 & 1.830 & 2.054 & 1.412 & 870 \\
Nav. & 557 & 115 & 442 & 677 & 860 & 938 & 1.016 & 989 & 1.513 & 1.300 \\
P.V. & -6.901 & -8.499 & -10.999 & -9.576 & -6.539 & -6.212 & -5.888 & -5.315 & -6.371 & -5.732 \\
Rioja & 572 & 470 & 517 & -72 & 549 & 126 & -293 & -274 & 209 & 177 \\
\hline
\end{tabular}


distancias cada vez más cortas. Nosotros nos centramos en los flujos entre comunidades autónomas.

El número de migraciones intrarregionales sufre la misma variación anual que las migraciones interregionales (gráfico $3 a$ ), salvo para los dos últimos años. Si consideramos las tasas correspondientes (migración entre población), se da la misma estructura (gráfico 3b), si bien las diferencias entre una y otras son más acusadas que en $a$ ) para los últimos años, indicando que las migraciones se realizan principalmente dentro de la misma comunidad autónoma.

Si nos fijamos en la migración neta por comunidades autónomas (tabla 4), Asturias, Castilla-León y País Vasco son siempre regiones emigrantes, mientras que son inmigrantes Comunidad Valenciana, Murcia y Navarra. Por otra parte, Aragón, Cantabria y Castilla la Mancha, pasan de ser regiones emigrantes a ser regiones inmigrantes (si bien cada una de ellas realiza el cambio en un año diferente). Madrid pasa de ser atractiva a perder población a través de la migración a partir de 1991. Otras regiones, como Baleares y Canarias, son siempre regiones atractivas salvo para un año concreto. En el resto de las comunidades autónomas, el signo de la migración neta cambia más de una vez dentro de este período.

En los tres primeros años, es Canarias la región con mayor migración neta, seguida casi siempre por Madrid. En 1988 y 1989 son Madrid y la Comunidad Valenciana las que más migrantes ganan. Pero Madrid, desde 1993, pasa a ser la comunidad autónoma que pierde mayor población con la migración. En años anteriores (salvo en 1993), era siempre el País Vasco.

Para examinar más detenidamente estos datos, calculamos la tasa de migración neta anual (tabla 5), definida como el cociente entre la migración neta y la población al principio del periodo.

Sigue siendo el País Vasco la comunidad autónoma con menor tasa de migración neta (salvo para 1989 y 1990). En el extremo opuesto se sitúan Baleares y Canarias. En cuanto a los diferentes valores que toma la tasa en los distintos años, hay que resaltar el hecho de que el rango de variación de la misma pasa de 7 en 1986 a casi 12 en 1987 (tabla 5), y sigue aumentando hasta 1990, año en el que comienza una tendencia descendente importante, debido a la disminución de la tasa de migración neta positiva (se compensan más las salidas con las entradas).

Distinguiendo entre tasa de emigración y de inmigración ${ }^{15}$ (tablas 6 y 7 , respectivamente), para las migraciones interregionales, se puede comprobar que las regiones con más (menos) inmigrantes son también las que tienen más (menos) emigrantes, destacando Baleares y La Rioja (Galicia y Asturias).

Mediante el gráfico de dispersión por años (gráfico 4), estudiamos cómo se comportan la emigración y la inmigración, conjuntamente. Si los puntos

15. Calculamos las tasas anuales de emigración e inmigración de forma análoga a la tasa de migración neta anual. 
Tabla 5. Tasa de migración neta por comunidades autónomas (en tanto por mil).

\begin{tabular}{lrrrrrrrrrr}
\hline & 1986 & 1987 & 1988 & 1989 & 1990 & 1991 & 1992 & 1993 & 1994 & 1995 \\
\hline And. & 0,269 & $-0,117$ & $-0,390$ & $-0,189$ & 0,235 & 0,324 & 0,404 & 0,650 & 0,357 & $-0,382$ \\
Arg. & $-0,032$ & $-0,261$ & 0,122 & 0,208 & 0,472 & 0,478 & 0,479 & 0,411 & 0,352 & 0,225 \\
Ast. & $-0,602$ & $-0,669$ & $-1,148$ & $-0,734$ & $-0,480$ & $-0,308$ & $-0,116$ & $-0,166$ & $-1,057$ & $-0,917$ \\
Bal. & 2,104 & 7,625 & 12,833 & 9,542 & 1,610 & 1,299 & 0,837 & $-0,482$ & 2,648 & 6,020 \\
Can. & 3,910 & 5,887 & 6,513 & 3,885 & $-1,015$ & 0,355 & 1,763 & 1,816 & 3,242 & 4,183 \\
Cant. & $-0,318$ & $-0,482$ & $-0,494$ & 0,280 & 0,108 & 0,173 & 0,232 & 0,944 & 1,629 & 1,415 \\
C.L. & $-1,294$ & $-2,092$ & $-2,574$ & $-3,544$ & $-3,315$ & $-2,643$ & $-1,882$ & $-1,220$ & $-0,466$ & $-1,185$ \\
C.M. & $-1,125$ & $-2,536$ & $-2,951$ & $-3,172$ & $-1,008$ & 0,424 & 1,862 & 2,266 & 3,112 & 3,101 \\
Cat. & $-0,911$ & $-0,286$ & 0,217 & 0,350 & 0,352 & $-0,191$ & $-0,737$ & $-1,008$ & $-1,169$ & $-0,570$ \\
C.V. & 0,915 & 0,709 & 1,522 & 2,175 & 1,935 & 1,420 & 0,875 & 1,122 & 1,400 & 1,836 \\
Ext. & $-0,438$ & $-1,474$ & $-4,791$ & $-5,804$ & $-4,095$ & $-2,342$ & $-0,435$ & 0,901 & 1,246 & $-0,422$ \\
Gal. & $-0,462$ & $-0,925$ & $-1,326$ & $-0,987$ & $-0,410$ & $-0,101$ & 0,234 & 0,411 & 0,268 & $-0,133$ \\
Mad. & 0,992 & 1,587 & 1,563 & 1,753 & 1,780 & 0,829 & $-0,150$ & $-1,266$ & $-2,203$ & $-2,067$ \\
Mur. & 1,947 & 1,260 & 0,855 & 2,053 & 1,092 & 1,428 & 1,737 & 1,900 & 1,284 & 0,784 \\
Nav. & 1,080 & 0,222 & 0,849 & 1,292 & 1,631 & 1,806 & 1,946 & 1,870 & 2,840 & 2,425 \\
P.V. & $-3,231$ & $-3,967$ & $-5,102$ & $-4,438$ & $-3,028$ & $-2,952$ & $-2,787$ & $-2,498$ & $-2,990$ & $-2,690$ \\
Rioja & 2,200 & 1,801 & 1,961 & $-0,271$ & 2,062 & 0,478 & $-1,109$ & $-1,030$ & 0,782 & 0,660 \\
\hline Max. & 3,910 & 7,625 & 12,833 & 9,542 & 2,062 & 1,806 & 1,946 & 2,266 & 3,242 & 6,020 \\
Min. & $-3,231$ & $-3,967$ & $-5,102$ & $-5,804$ & $-4,095$ & $-2,952$ & $-2,787$ & $-2,498$ & $-2,990$ & $-2,690$ \\
Recor. & 7,141 & 11,592 & 17,935 & 15,346 & 6,157 & 4,759 & 4,733 & 4,763 & 6,233 & 8,711 \\
\hline
\end{tabular}

Máx. y Mín. representan, respectivamente, los valores máximo y mínimo de la tasa de migración. Recor. es la diferencia entre el valor máximo y el mínimo.
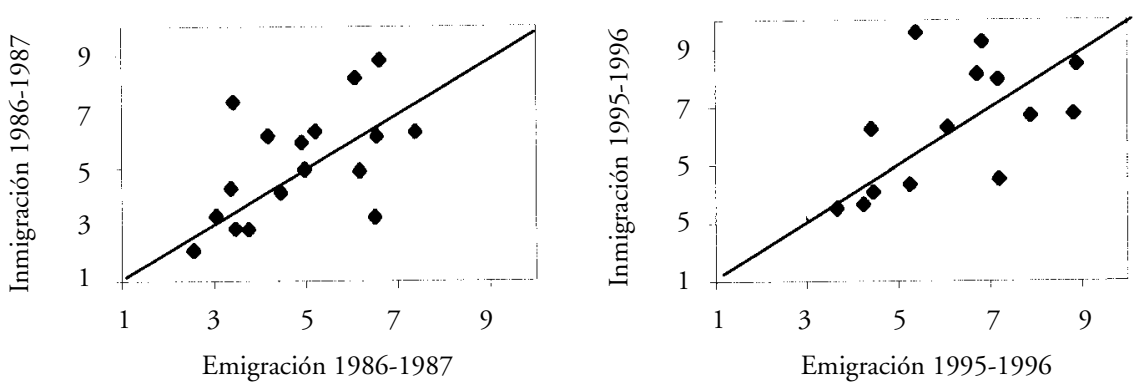

Gráfico 4. Tasa de inmigración frente a tasa de emigración.

están próximos a la diagonal, que representa tasas migratorias iguales, por unidad de población, se compensan la emigración y la inmigración. Si están alejados de la diagonal, nos informa de una fuerte polarización de los flujos hacia algunas regiones concretas (existen menos diferencias en 1986).

Con el fin de estudiar si existe alguna asociación entre la inmigración y la emigración, calculamos, para cada año, el coeficiente de correlación entre estas 
Tabla 6. Tasa de emigración por comunidades autónomas (en tanto por mil).

\begin{tabular}{lrrrrrrrrrr}
\hline & 1986 & 1987 & 1988 & 1989 & 1990 & 1991 & 1992 & 1993 & 1994 & 1995 \\
\hline And. & 3,049 & 3,447 & 4,556 & 5,116 & 4,701 & 4,296 & 3,751 & 3,609 & 3,856 & 4,448 \\
Arg. & 4,971 & 5,050 & 5,726 & 6,070 & 6,194 & 5,798 & 5,344 & 5,636 & 5,909 & 6,069 \\
Ast. & 3,472 & 3,444 & 4,428 & 5,043 & 4,880 & 4,588 & 4,126 & 4,382 & 5,179 & 5,249 \\
Bal. & 6,073 & 6,290 & 8,085 & 10,818 & 13,031 & 13,188 & 11,947 & 10,452 & 8,636 & 8,301 \\
Can. & 3,426 & 3,570 & 4,904 & 6,657 & 8,140 & 6,917 & 5,103 & 4,923 & 4,872 & 5,390 \\
Cant. & 4,454 & 4,678 & 5,380 & 6,263 & 5,979 & 5,630 & 5,187 & 5,277 & 5,904 & 6,721 \\
C.L. & 6,174 & 6,594 & 8,090 & 9,547 & 9,412 & 8,439 & 7,211 & 7,265 & 7,488 & 7,886 \\
C.M. & 7,384 & 8,384 & 10,390 & 11,624 & 10,770 & 9,485 & 7,904 & 8,338 & 8,866 & 9,497 \\
Cat. & 3,757 & 3,402 & 3,984 & 4,306 & 4,311 & 4,296 & 4,190 & 4,449 & 4,607 & 4,233 \\
C.V. & 3,378 & 3,597 & 4,046 & 4,427 & 4,382 & 4,278 & 4,090 & 4,116 & 4,316 & 4,406 \\
Ext. & 6,542 & 7,340 & 11,145 & 13,048 & 12,239 & 10,612 & 8,483 & 7,913 & 7,984 & 8,896 \\
Gal. & 2,556 & 2,826 & 3,925 & 4,105 & 3,893 & 3,511 & 2,863 & 2,892 & 3,274 & 3,653 \\
Mad. & 4,904 & 4,649 & 6,000 & 6,841 & 7,111 & 6,858 & 6,438 & 7,476 & 8,626 & 8,830 \\
Mur. & 4,176 & 4,586 & 5,414 & 6,061 & 6,556 & 6,020 & 5,348 & 5,581 & 6,608 & 7,174 \\
Nav. & 5,208 & 5,437 & 6,222 & 6,901 & 6,761 & 6,417 & 5,950 & 6,369 & 6,464 & 6,828 \\
P.V. & 6,502 & 6,954 & 8,365 & 8,544 & 7,425 & 7,001 & 6,359 & 6,444 & 7,328 & 7,199 \\
Rioja & 6,603 & 6,744 & 8,244 & 9,492 & 8,694 & 8,731 & 8,675 & 9,410 & 9,721 & 10,533 \\
\hline Máx. & 7,384 & 8,384 & 11,145 & 13,048 & 13,031 & 13,188 & 11,947 & 10,452 & 9,721 & 10,533 \\
Mín. & 2,556 & 2,826 & 3,925 & 4,105 & 3,893 & 3,511 & 2,863 & 2,892 & 3,274 & 3,653 \\
Recor. & 4,828 & 5,558 & 7,220 & 8,943 & 9,138 & 9,676 & 9,085 & 7,560 & 6,447 & 6,880 \\
\hline
\end{tabular}

Máx. y Mín. representan, respectivamente, los valores máximo y mínimo de la tasa de emigración; Recor. es la diferencia entre el valor máximo y el mínimo.

variables, así como sobre las tasas, incluyendo también la migración neta (tabla 8). La inmigración está muy correlacionada con la emigración, pero si tenemos en cuenta las tasas correspondientes, esta correlación no tiene lugar hasta 1990-1991, siendo la más alta para el periodo siguiente, 1991-1992. Por el contrario, la migración neta sólo está correlacionada, aunque débilmente y de forma negativa, con la emigración en los últimos años de nuestro estudio. Sin embargo, sí existe una asociación positiva más fuerte entre las tasas de migración neta y la de inmigración, en los cuatro primeros años y en los dos últimos, con el máximo valor de 0,86 para 1988-1989. Por tanto, la inmigración influye más en la tasa de migración neta que la emigración. Existe una amplia literatura sobre este tema, Plane et al. (1984) argumentan que, dado que la variabilidad de la inmigración es superior a la de emigración, la primera debe influir más en la migración neta.

Si calculamos la varianza regional de la tasa de emigración e inmigración para cada año (tabla 9), tenemos que la inmigración tiene mayor varianza que la emigración, a excepción de algún año, como 1990-1991, en el que se podrían considerar iguales. Ambas varianzas aumentan con el paso del tiempo, la inmigración con mayor intensidad, hasta 1990-1991, en el que se igualan, para disminuir posteriormente, aunque ahora es la emigración la que más cambia. En el último año ambas vuelven a crecer. 
Tabla 7. Tasa de inmigración por comunidades autónomas (en tanto por mil).

\begin{tabular}{lrrrrrrrrrr}
\hline & 1986 & 1987 & 1988 & 1989 & 1990 & 1991 & 1992 & 1993 & 1994 & 1995 \\
\hline And. & 3,318 & 3,330 & 4,166 & 4,927 & 4,936 & 4,620 & 4,155 & 4,260 & 4,214 & 4,066 \\
Arg. & 4,939 & 4,789 & 5,848 & 6,278 & 6,666 & 6,276 & 5,823 & 6,047 & 6,261 & 6,294 \\
Ast. & 2,870 & 2,775 & 3,281 & 4,309 & 4,400 & 4,280 & 4,010 & 4,216 & 4,122 & 4,332 \\
Bal. & 8,177 & 13,915 & 20,918 & 20,360 & 14,641 & 14,487 & 12,785 & 9,970 & 11,284 & 14,321 \\
Can. & 7,336 & 9,457 & 11,416 & 10,542 & 7,125 & 7,272 & 6,865 & 6,739 & 8,114 & 9,573 \\
Cant. & 4,137 & 4,196 & 4,886 & 6,544 & 6,088 & 5,803 & 5,419 & 6,222 & 7,533 & 8,136 \\
C.L. & 4,880 & 4,501 & 5,515 & 6,003 & 6,097 & 5,796 & 5,329 & 6,044 & 7,022 & 6,701 \\
C.M. & 6,259 & 5,847 & 7,440 & 8,452 & 9,761 & 9,909 & 9,766 & 10,604 & 11,978 & 12,598 \\
Cat. & 2,845 & 3,116 & 4,202 & 4,656 & 4,663 & 4,105 & 3,453 & 3,441 & 3,438 & 3,663 \\
C.V. & 4,293 & 4,306 & 5,568 & 6,602 & 6,317 & 5,699 & 4,965 & 5,238 & 5,715 & 6,242 \\
Ext. & 6,104 & 5,866 & 6,354 & 7,244 & 8,144 & 8,270 & 8,049 & 8,815 & 9,230 & 8,474 \\
Gal. & 2,094 & 1,901 & 2,599 & 3,118 & 3,483 & 3,410 & 3,097 & 3,303 & 3,542 & 3,520 \\
Mad. & 5,896 & 6,236 & 7,563 & 8,594 & 8,890 & 7,688 & 6,289 & 6,210 & 6,423 & 6,763 \\
Mur. & 6,122 & 5,845 & 6,269 & 8,114 & 7,648 & 7,448 & 7,086 & 7,481 & 7,893 & 7,958 \\
Nav. & 6,288 & 5,659 & 7,071 & 8,193 & 8,392 & 8,223 & 7,896 & 8,239 & 9,303 & 9,252 \\
P.V. & 3,271 & 2,987 & 3,263 & 4,106 & 4,397 & 4,049 & 3,572 & 3,946 & 4,338 & 4,509 \\
Rioja & 8,803 & 8,545 & 10,205 & 9,221 & 10,755 & 9,209 & 7,566 & 8,380 & 10,503 & 11,193 \\
\hline Máx. & 8,803 & 13,915 & 20,918 & 20,360 & 14,641 & 14,487 & 12,785 & 10,604 & 11,978 & 14,321 \\
Mín. & 2,094 & 1,901 & 2,599 & 3,118 & 3,483 & 3,410 & 3,097 & 3,303 & 3,438 & 3,520 \\
Recor. & 6,709 & 12,014 & 18,319 & 17,242 & 11,158 & 11,077 & 9,688 & 7,301 & 8,540 & 10,801 \\
\hline
\end{tabular}

Máx. y Mín. representan, respectivamente, los valores máximo y mínimo de la tasa de inmigración. Recor. es la diferencia entre el valor máximo y el mínimo.

Tabla 8. Correlación entre las tasas.

\begin{tabular}{lcccccc}
\hline & \multirow{2}{*}{$\begin{array}{c}\text { I } \\
\text { frente a }\end{array}$} & \multicolumn{2}{c}{$\begin{array}{c}\text { MN } \\
\text { frente a }\end{array}$} & \multicolumn{2}{c}{ TI } & \multicolumn{2}{c}{ TMN } \\
frente a & \multicolumn{2}{c}{ frente a } \\
Año & E & I & E & TE & TI & TE \\
\hline $1986-1987$ & 0,901 & 0,252 & $-0,192$ & 0,550 & 0,678 & $-0,240$ \\
$1987-1988$ & 0,839 & 0,395 & $-0,168$ & 0,345 & 0,834 & $-0,230$ \\
$1988-1989$ & 0,832 & 0,409 & $-0,166$ & 0,313 & 0,860 & $-0,215$ \\
$1989-1990$ & 0,877 & 0,426 & $-0,060$ & 0,480 & 0,736 & $-0,241$ \\
$1990-1991$ & 0,936 & 0,536 & 0,205 & 0,768 & 0,354 & $-0,327$ \\
$1991-1992$ & 0,956 & 0,375 & 0,086 & 0,862 & 0,372 & $-0,151$ \\
$1992-1993$ & 0,952 & 0,071 & $-0,236$ & 0,852 & 0,449 & $-0,086$ \\
$1993-1994$ & 0,939 & 0,109 & $-0,444$ & 0,805 & 0,406 & $-0,216$ \\
$1994-1995$ & 0,920 & $-0,245$ & $-0,604$ & 0,743 & 0,704 & 0,047 \\
$1995-1996$ & 0,925 & $-0,252$ & $-0,601$ & 0,706 & 0,769 & 0,090 \\
\hline
\end{tabular}

Nota: $\mathrm{I}$ = inmigración; $\mathrm{E}$ = emigración; $\mathrm{MN}$ = migración neta; $\mathrm{TI}$ = tasa de inmigración; $\mathrm{TE}=$ tasa de emigración; TMN = tasa de migración neta. 
Por otro lado, si un año hay mucha inmigración, ¿significa que en el siguiente va a continuar siendo alta? Para responder a esta pregunta, obtenemos el coeficiente de correlación entre un año y el siguiente, para cada una de las tasas (migración neta, inmigración y emigración). Los resultados (tabla 10), muestran la alta correlación existente en todas las tasas, siendo siempre menor en la tasa de migración neta, con un mínimo entre 1989-1990 y 1990-1991 de 0,715.

Otra forma de estudiar los movimientos migratorios es a través del índice de efectividad, definido en el epígrafe 2, que nos indica como evolucionan los flujos, sin que esta medida dependa de la población de la región. El índice de efectividad global (6) presenta una tendencia creciente en los tres primeros años (tabla 11), debido fundamentalmente al mayor aumento de la migración neta sobre la migración total. Nos indica que aunque la movilidad ha aumentado, se ha producido un cambio en los modelos de elección de las regiones. En 1989 el índice disminuye, pues crece considerablemente el número de movimientos y la migración neta se mantiene prácticamente igual a la del año anterior. Podríamos afirmar que se mantienen las mismas elecciones que en 1988, pero con una mayor movilidad de la población. Continúa el descenso hasta

Tabla 9. Varianza regional de TI (VTI) y TE (VTE).

\begin{tabular}{lrlc}
\hline Año & VTI & VTE & VTI/VTE \\
\hline $1986-1987$ & 3,579 & 2,052 & 1,744 \\
$1987-1988$ & 8,141 & 2,616 & 3,112 \\
$1988-1989$ & 17,511 & 4,769 & 3,671 \\
$1989-1990$ & 14,218 & 6,922 & 2,054 \\
$1990-1991$ & 7,325 & 7,171 & 1,022 \\
$1991-1992$ & 7,120 & 6,278 & 1,134 \\
$1992-1993$ & 5,973 & 4,806 & 1,243 \\
$1993-1994$ & 4,718 & 4,132 & 1,142 \\
$1994-1995$ & 6,947 & 3,514 & 1,977 \\
$1995-1996$ & 9,449 & 3,890 & 2,429 \\
\hline
\end{tabular}

$\mathrm{TI}=$ tasa de inmigración; $\mathrm{TE}=$ tasa de emigración.

Tabla 10. Correlación regional de las tasas.

\begin{tabular}{llllllllll}
\hline & $\begin{array}{l}1986-87 \\
\text { frente a }\end{array}$ & $\begin{array}{l}1987-88 \\
\text { frente a }\end{array}$ & $\begin{array}{l}1988-89 \\
\text { frente a }\end{array}$ & $\begin{array}{l}1989-90 \\
\text { frente a }\end{array}$ & $\begin{array}{l}1990-91 \\
\text { frente a }\end{array}$ & $\begin{array}{l}1991-92 \\
\text { frente a }\end{array}$ & $\begin{array}{l}1992-93 \\
\text { frente a }\end{array}$ & $\begin{array}{l}1993-94 \\
\text { frente a }\end{array}$ & $\begin{array}{l}1994-95 \\
\text { frente a }\end{array}$ \\
& $1987-88$ & $1988-89$ & $1989-90$ & $1990-91$ & $1991-92$ & $1992-93$ & $1993-94$ & $1994-95$ & $1995-96$ \\
\hline TE & 0,985 & 0,964 & 0,977 & 0,954 & 0,985 & 0,977 & 0,972 & 0,951 & 0,982 \\
TI & 0,885 & 0,982 & 0,987 & 0,915 & 0,987 & 0,987 & 0,945 & 0,985 & 0,971 \\
TMN & 0,871 & 0,967 & 0,959 & 0,715 & 0,909 & 0,794 & 0,894 & 0,818 & 0,890 \\
IND & 0,925 & 0,968 & 0,950 & 0,794 & 0,923 & 0,852 & 0,933 & 0,880 & 0,914 \\
\hline
\end{tabular}

$\mathrm{TE}=$ tasa de emigración $; \mathrm{TI}=$ tasa de inmigración; $\mathrm{TMN}$ = tasa de migración neta; IND = índice de efectividad. 
1991-1992, y se produce en 1990 la bajada más importante, debido a que se mantiene el número total de migraciones, pero la migración neta disminuye en 21.800 migrantes, hay un intercambio entre emigración e inmigración. Desde 1992 hasta 1994 el índice crece, bien porque disminuye la migración total, como en 1992, o bien porque, aunque aumenta la movilidad, la migración neta lo hace en mayor proporción. En 1995 hay una pequeña bajada del índice, por un proceso contrario al de 1992, aumenta la migración total y la migración neta cambia poco.

A partir de la correlación del índice de efectividad para períodos distintos, podemos conocer si se ha producido o no un cambio en los modelos de redistribución de la población. Una correlación positiva fuerte significa que la distribución de la eficiencia es bastante similar, es decir, que hay estabilidad. Una correlación baja indica ajustes en dicha estructura. Una relación negativa es síntoma de un cambio generalizado de las corrientes y contracorrientes de la migración. En España, para el período 1986-1995 (última fila de la tabla 10), entre el índice de un año y el del año anterior existe una correlación positiva fuerte (con cualquier otro año también se cumple, pero va disminuyendo conforme aumenta el período de comparación). Cabe destacar que la correlación menor se da entre 1989 y 1990, y es, por tanto, 1990, el año en el que los cambios migratorios son más acusados.

El índice de efectividad no está correlacionado con la tasa de inmigración y emigración, pero sí y muy fuerte con la tasa de migración neta (tabla 12). Si a esto unimos el hecho de no tener el problema que existe en la definición de las tasas, en cuanto a qué población es la más adecuada como cociente, hace que el índice de efectividad sea una medida atractiva.

La descomposición de los cambios en el índice (tabla 13), a través de sus distintas posibilidades en relación con las diversas comunidades autónomas (4), revela la naturaleza compleja del concepto de índice. Los cambios más evidentes son aquellos relacionados con el cambio de inmigración a emigración,

Tabla 11. Índice de efectividad.

\begin{tabular}{llll}
\hline Año & $\begin{array}{l}\text { Migración neta } \\
\text { (en valor absoluto) }\end{array}$ & $\begin{array}{l}\text { Migración } \\
\text { total }\end{array}$ & Índice \\
\hline $1986-1987$ & 40.476 & 165.645 & 24,435 \\
$1987-1988$ & 52.524 & 173.401 & 30,290 \\
$1988-1989$ & 72.088 & 219.998 & 32,768 \\
$1989-1990$ & 71.332 & 249.828 & 28,552 \\
$1990-1991$ & 49.532 & 248.448 & 19,937 \\
$1991-1992$ & 34.400 & 225.582 & 15,249 \\
$1992-1993$ & 33.618 & 202.852 & 16,573 \\
$1993-1994$ & 43.864 & 212.725 & 20,620 \\
$1994-1995$ & 54.726 & 230.334 & 23,759 \\
$1995-1996$ & 55.430 & 241.279 & 22,973 \\
\hline
\end{tabular}


o viceversa. La medida del índice puede aumentar o disminuir, incluso aunque la dirección neta de migrantes no cambie. La evolución temporal del índice está reflejada en el gráfico 5 .

Sin tener en cuenta el signo, Canarias y País Vasco presentan, en 1986, los mayores índices de efectividad, superiores al 20\%, lo que indica que la inmi-

Tabla 12. Correlación de las tasas con el índice de efectividad.

\begin{tabular}{lccc}
\hline & \multicolumn{3}{c}{$\begin{array}{c}\text { Índice de efectividad } \\
\text { frente }\end{array}$} \\
\cline { 2 - 4 } Periodo & TI & TE & TMN \\
\hline $1986-1987$ & 0,068 & $-0,332$ & 0,983 \\
$1987-1988$ & 0,256 & $-0,275$ & 0,958 \\
$1988-1989$ & 0,308 & $-0,243$ & 0,932 \\
$1989-1990$ & 0,277 & $-0,190$ & 0,936 \\
$1990-1991$ & 0,249 & $-0,082$ & 0,968 \\
$1991-1992$ & 0,142 & $-0,138$ & 0,974 \\
$1992-1993$ & 0,014 & $-0,265$ & 0,977 \\
$1993-1994$ & $-0,093$ & $-0,374$ & 0,968 \\
$1994-1995$ & $-0,190$ & $-0,504$ & 0,971 \\
$1995-1996$ & $-0,124$ & $-0,448$ & 0,965 \\
\hline
\end{tabular}

Nota: $\mathrm{TI}=$ tasa de inmigración $; \mathrm{TE}=$ tasa de emigración; $\mathrm{TMN}=$ tasa de migración neta.

Tabla 13. Índice de efectividad por comunidades autónomas, para el período 1986-1987 hasta 1995-1996.

\begin{tabular}{lrrrrrrrrrrr}
\hline CC.AA. 1986 & 1987 & 1988 & 1989 & 1990 & 1991 & 1992 & 1993 & 1994 & 1995 & Cambio* \\
\hline And. & 4,219 & $-1,725$ & $-4,471$ & $-1,883$ & 2,438 & 3,634 & 5,113 & 8,266 & 4,429 & $-4,488$ & $-8,707$ \\
Arg. & $-0,324$ & $-2,648$ & 1,057 & 1,686 & 3,670 & 3,957 & 4,285 & 3,520 & 2,893 & 1,818 & 2,142 \\
Ast. & $-9,498$ & $-10,759$ & $-14,886$ & $-7,849$ & $-5,176$ & $-3,474$ & $-1,431$ & $-1,934$ & $-11,370$ & $-9,575$ & $-0,077$ \\
Bal. & 14,769 & 37,739 & 44,249 & 30,606 & 5,816 & 4,693 & 3,385 & $-2,362$ & 13,295 & 26,613 & 11,844 \\
Can. & 36,333 & 45,190 & 39,907 & 22,588 & $-6,652$ & 2,500 & 14,728 & 15,574 & 24,969 & 27,953 & $-8,379$ \\
Cant. & $-3,697$ & $-5,433$ & $-4,814$ & 2,188 & 0,899 & 1,509 & 2,189 & 8,213 & 12,120 & 9,527 & 13,224 \\
C.L. & $-11,704$ & $-18,858$ & $-18,920$ & $-22,790$ & $-21,373$ & $-18,568$ & $-15,009$ & $-9,168$ & $-3,214$ & $-8,122$ & 3,581 \\
C.M. & $-8,245$ & $-17,823$ & $-16,550$ & $-15,801$ & $-4,910$ & 2,186 & 10,538 & 11,961 & 14,929 & 14,035 & 22,281 \\
Cat. & $-13,802$ & $-4,382$ & 2,655 & 3,902 & 3,920 & $-2,275$ & $-9,649$ & $-12,779$ & $-14,533$ & $-7,217$ & 6,585 \\
C.V. & 11,931 & 8,966 & 15,830 & 19,717 & 18,084 & 14,234 & 9,662 & 11,996 & 13,954 & 17,239 & 5,308 \\
Ext. & $-3,465$ & $-11,160$ & $-27,380$ & $-28,604$ & $-20,091$ & $-12,405$ & $-2,629$ & 5,387 & 7,238 & $-2,427$ & 1,038 \\
Gal. & $-9,927$ & $-19,571$ & $-20,319$ & $-13,660$ & $-5,559$ & $-1,465$ & 3,930 & 6,640 & 3,927 & $-1,851$ & 8,076 \\
Mad. & 9,184 & 14,579 & 11,528 & 11,356 & 11,122 & 5,701 & $-1,175$ & $-9,253$ & $-14,639$ & $-13,26$ & $-22,443$ \\
Mur. & 18,904 & 12,076 & 7,322 & 14,486 & 7,689 & 10,601 & 13,972 & 14,547 & 8,855 & 5,180 & $-13,725$ \\
Nav. & 9,391 & 2,005 & 6,385 & 8,560 & 10,763 & 12,339 & 14,053 & 12,803 & 18,010 & 15,078 & 5,686 \\
P.V. & $-33,056$ & $-39,903$ & $-43,878$ & $-35,085$ & $-25,610$ & $-26,718$ & $-28,065$ & $-24,038$ & $-25,635$ & $-22,98$ & 10,080 \\
Rioja & 14,279 & 11,779 & 10,627 & $-1,450$ & 10,601 & 2,666 & $-6,828$ & $-5,788$ & 3,868 & 3,038 & $-11,241$ \\
\hline
\end{tabular}

* «Cambio» significa la diferencia entre el valor del índice de 1995 y el de 1986. 
Tabla 14. No de comunidades autónomas según el valor del índice de efectividad.

\begin{tabular}{cccc}
\hline Variación & $\mathbf{1 9 8 6 - 1 9 8 7}$ & $\mathbf{1 9 9 5 - 1 9 9 6}$ & Cambio \\
\hline$>20 \%$ & 1 & 2 & 1 \\
$10 \%-20 \%$ & 4 & 3 & 3 \\
$0 \%-10 \%$ & 3 & 4 & 7 \\
$-10 \%-0 \%$ & 6 & 6 & 3 \\
$-20 \%--10 \%$ & 2 & 1 & 2 \\
$<-20 \%$ & 1 & 1 & 1 \\
\hline
\end{tabular}

gración (emigración) es 1,5 veces superior a la emigración (inmigración) si el índice es positivo (negativo). Les siguen Baleares, Castilla León, Cataluña, Comundiad Valenciana, Murcia y La Rioja, con valores entre el $10 \%$ y el $20 \%$ (inmigración y emigración) 1,22 veces superior a la emigración (inmigración), si el signo es positivo (negativo). Del resto de las comunidades autónomas, tres tienen un índice entre 0 y $10 \%$ y seis entre $-10 \%$ y 0 . Por años, el índice varía desde -33.056 para el País Vasco, hasta 36.333 para Canarias, en 1986, con un rango de 69.388, el mismo aumentó hasta 1988 y disminuyó considerablemente hasta 40.793 en 1991, siendo la Comunidad Valenciana (14.234) y el País Vasco (-6.718) las regiones con los valores extremos; lo cual indica que los movimientos son menos unidireccionales con el paso del tiempo. El rango del índice vuelve a aumentar en 1992, disminuye en 1993 y aumenta durante los dos últimos años. Hay dos comunidades autónomas (Baleares y Canarias), en 1995, con un índice positivo superior al 20\%, hay siete en el intervalo $[0,20]$, seis en el $[-10,0]$ y dos (Madrid y País Vasco) inferior al $-10 \%$.

En la tabla 14 podemos observar el cambio absoluto en el índice durante el período 1986-1995. En once comunidades autónomas aumenta y en seis disminuye (Andalucía, Asturias, Canarias, Madrid, Murcia y La Rioja). En todos los que disminuye (aumenta) las variaciones en la emigración (inmigración) son mayores en magnitud que las de la inmigración (emigración). En aquellas regiones en las que disminuye (aumenta) el índice, se produce una variación negativa (positiva) en la migración neta, salvo para Canarias.

A partir del índice entre cada par de comunidades autónomas calculado mediante (5), se puede estudiar la correlación que existe entre el primer y el último año, y ello da una medida de la estabilidad de la migración de esa región con el resto (tabla 15). Si es positiva y alta indica eficiencia estable (caso de Andalucía, Castilla y León, Comunidad Valenciana, Galicia y Madrid), mientras que si es negativa y alta significa que se ha producido un cambio total (no hay ninguna con estas características). Una baja correlación nos dice que la eficiencia con el resto de las regiones es bastante diferente, ha ganado y ha perdido migrantes para un conjunto diferente de estados en cada período.

Si estudiamos el índice entre cada par de comunidades autónomas, podemos averiguar hacia donde se mueven y de donde vienen los migrantes, de cada comunidad autónoma. Por ejemplo, en el gráfico 6 se observan algunas de estas direcciones, durante el primer y último año de estudio, para Andalucía. 
1986-1987
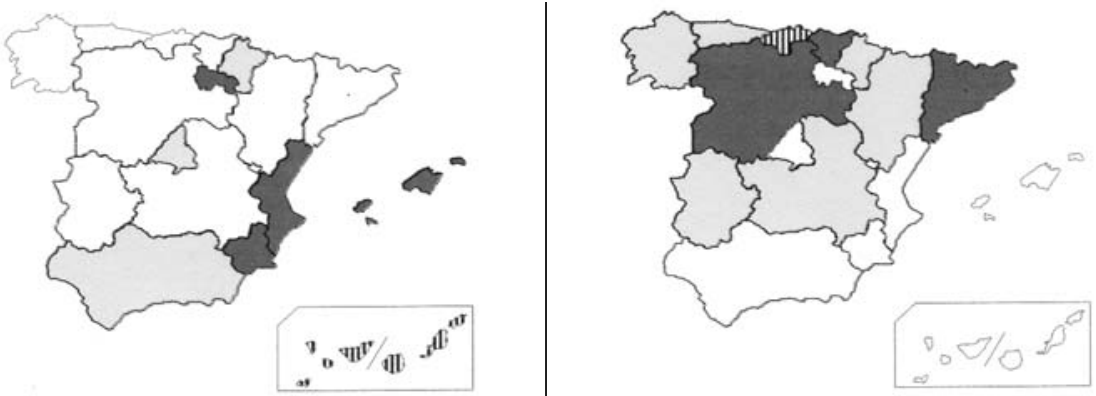

1987-1988
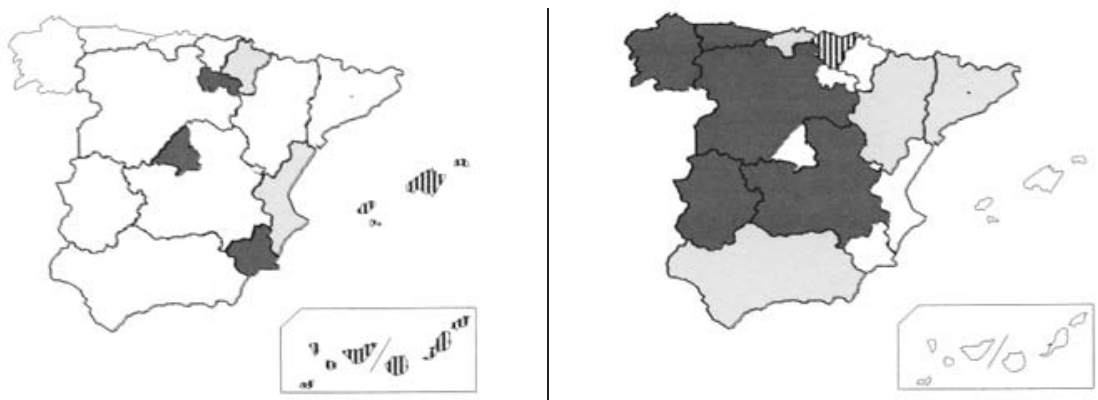

1988-1989
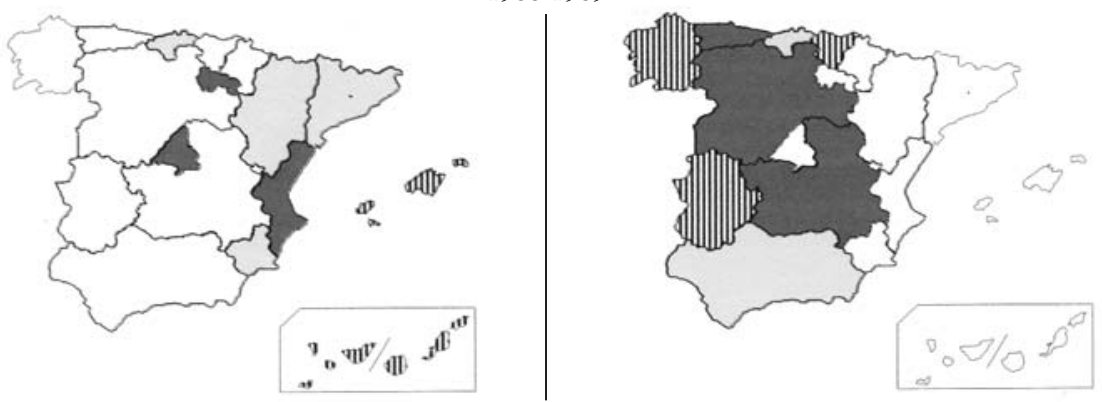

Índices (\%)

Índices (\%)
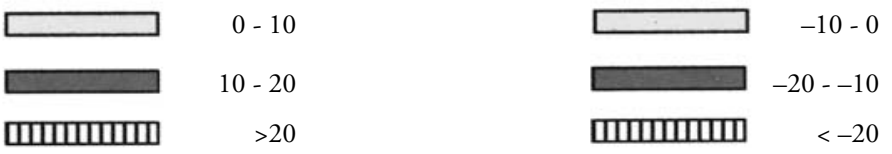

Gráfico 5. Índices de efectividad anuales. 

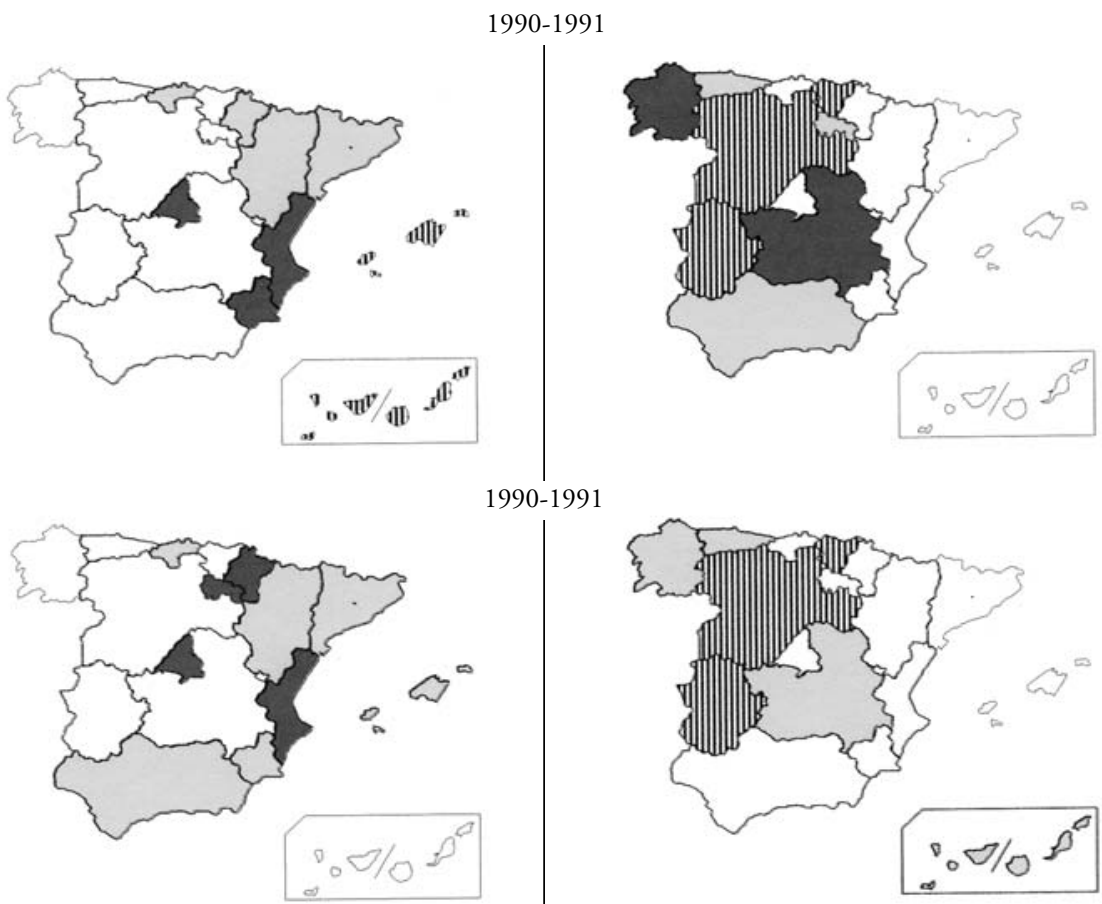

1990-1991

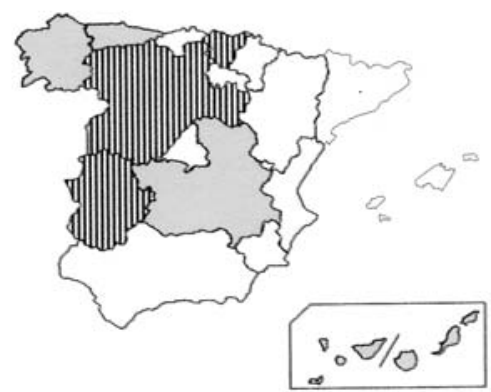

1991-1992

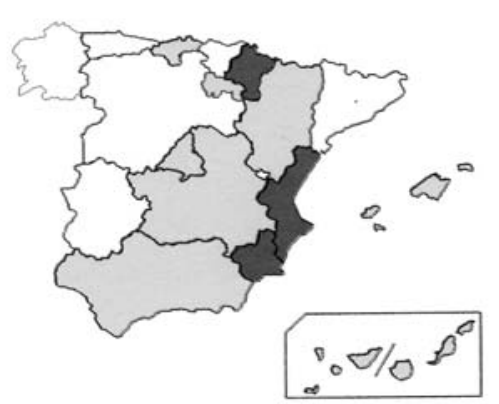

Índices (\%)

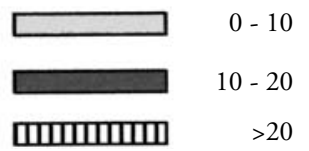

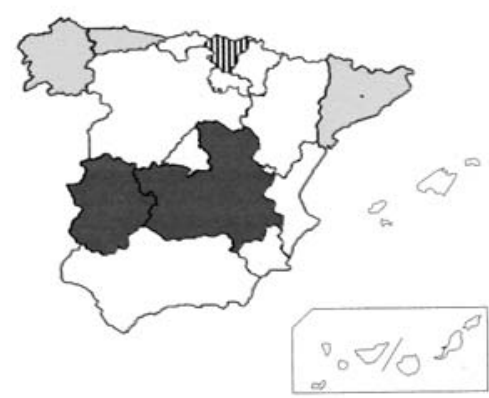

Índices (\%)

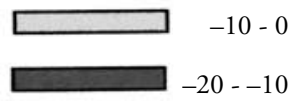

IIIIIIII) $<-20$

Gráfico 5. Índices de efectividad anuales (continuación). 
1993-1994
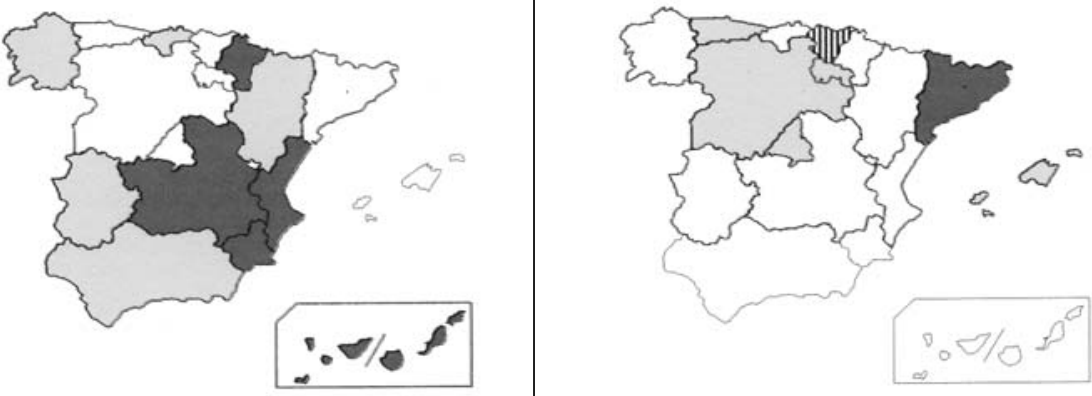

1994-1995
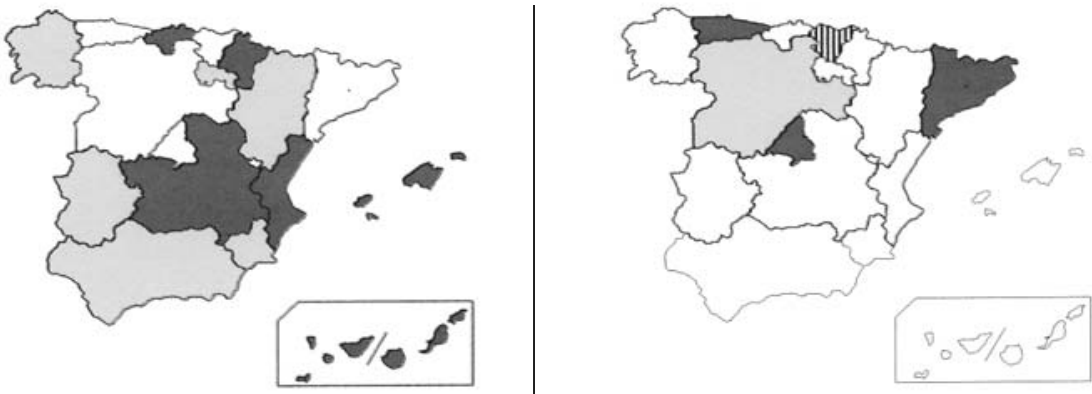

1995-1996
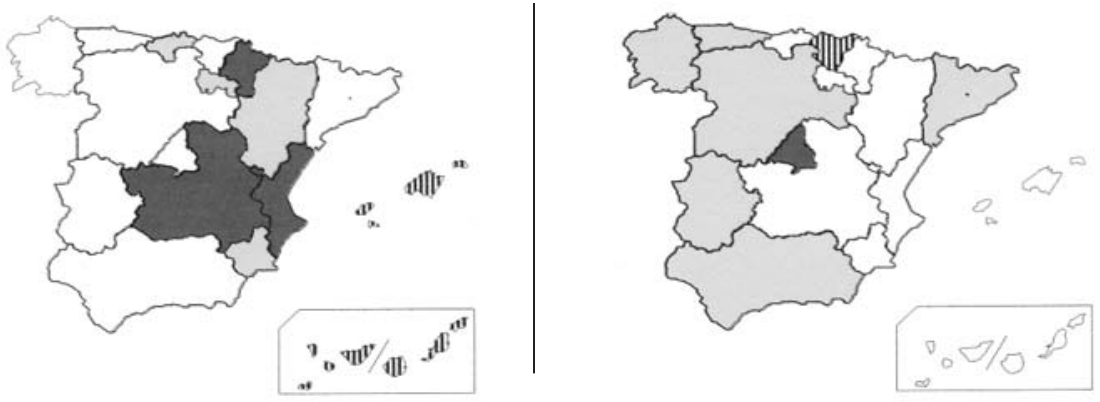

Índices (\%)

Índices (\%)
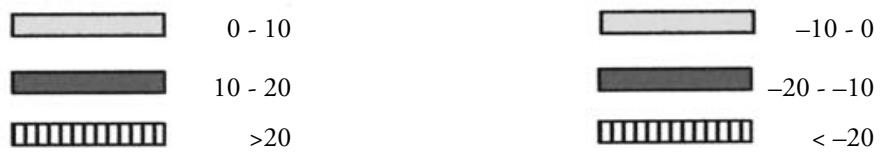

Gráfico 5. Índices de efectividad anuales (continuación). 
Tabla 15. Correlación del índice de efectividad de 1986-1987 frente a 1995-1996, para cada comunidad autónoma.

\begin{tabular}{ll}
\hline Andalucía & $0,759^{* *}$ \\
Aragón & 0,484 \\
Asturias & $0,569^{*}$ \\
Baleares & $0,682^{* *}$ \\
Canarias & 0,439 \\
Cantabria & $0,680^{* *}$ \\
Castilla y León & $0,826^{* *}$ \\
Castilla la Mancha & $0,570^{*}$ \\
Cataluña & $0,682^{* *}$ \\
Comunidad Valenciana & $0,794^{* *}$ \\
Extremadura & $0,578^{*}$ \\
Galicia & $0,871^{* *}$ \\
Madrid & $0,740^{* *}$ \\
Murcia & $0,516^{*}$ \\
Navarra & 0,191 \\
País Vasco & 0,449 \\
Rioja (La) & 0,207 \\
\hline
\end{tabular}

* Significativo al nivel 0,01.

** Significativo al nivel 0,05 .

a)

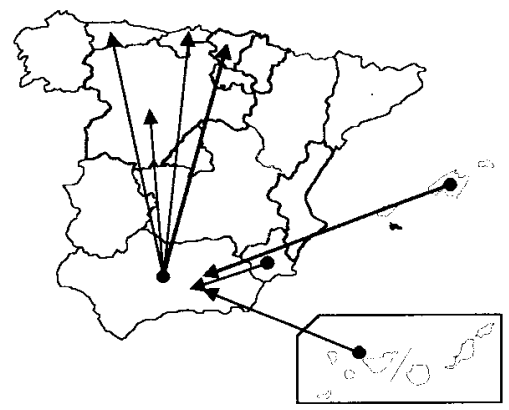

b)

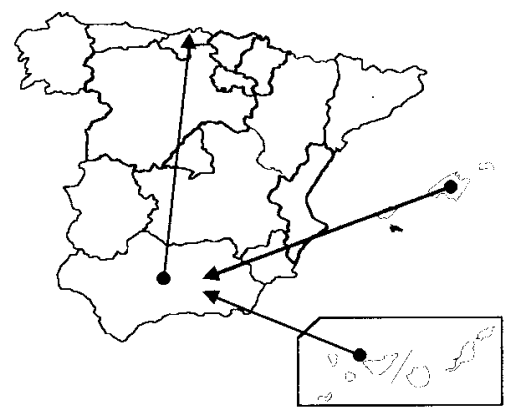

Gráfico 6. Índice de efectividad de Andalucía con el resto de las comunidades autónomas: a) 1986 ; b) 1995 . 
En concreto, reflejamos los intercambios de migración superiores al 20\% (en valor absoluto) del índice de efectividad. Durante el primer año hay siete corrientes con estas características, de las cuales tres eran positivas, correspondientes a regiones próximas. Sin embargo, en 1995 sólo hay tres corrientes con estas características, una de las cuales es inmigratoria.

\section{Bibliografía}

BEAMAN, J.; D’ARCY, C. (1980). «A Typology of Internal Migration». Canadian Studies in Population, vol. 7, p. 9-20.

Bentolila, S.; Dolado, J.J. (1990). «Mismatch and Internal Migration in Spain». Banco de España, Documento de trabajo, núm. 9006.

Bogue, D.J. (1959). «Internal Migration». En Hauser, P.M.; Duncan, O.D. (eds.). The Study of Population. Chicago, p. 486-509.

Brown, L.A.; MoOre, E.G. (1968). Intra-Urban Migration. Mimeo. Universidad de Iowa, Departamento de Geografía.

CABRÉ, A.; MORENO, J.; PUJADAS, I. (1985). «Cambio Migratorio y Reconversión Territorial en España». Revista Española de Investigaciones Sociológicas, 32, p. 43-65.

Courgeau, D. (1988). Méthodes de Mesure de la Mobilité Spatiale. París: INED.

Goldscheider, C. (1971). Population, Modernization and Social Structure. Boston: Little, Brown and Company.

HAENSZEL, W. (1967). "Concept, Measurement, and Data in Migration Analysis». Demography, vol. 4, p. 253-261.

Hamilton, C.H. (1965). «Practical and Mathematical Considerations in the Formulation and Selection of Migration Rates». Demography, vol. 2, núm. 3, p. 429-443.

- (1966). «Effect of Census Errors on the Measurement of Net Migration». Demography, vol. 3, núm. 3, p. 393-415.

- (1967). «The Vital Statistics Method of Estimating Net Migration by Age Cohorts». Demography, vol. 4, p. 464-478.

KinTNER, H.J.; Swanson, D.A. (1993). «Measurement Errors in Census Counts and Estimates of Intercensal Net Migration». Journal of Economics and Social Measurement, vol. 19, p. 97-120.

LeE, E.S. (1966). "A Theory of Migration». Demography, vol. 3, núm. 1, p. 45-57. Leguina Herrán, J.; FernándeZ Cordón, A. (1991): «Situación y Perspectivas de la Población Española». Economía y Sociología del Trabajo, 11, p. 52-60.

MCFARLAND, D.D. (1970). «Intragenerational Social Mobility as a Markov Process: Including a Time-Stationary Markovian Model that Explains Observed Declines in Mobility Rates over Time». American Sociological Review, vol. 35, p. 463-475.

McGinNiS, R. (1968). "A Stochastic Model Social Mobility». American Sociological Review, vol. 33, núm. 5, p. 712-721.

Musgrove, P. (1963). The Migration Elite. Londres: Heinemann.

Nurun Nabi, A.K.M.; Krishnan, P. (1993). «Some Approaches to the Study of Human Migration». En Mahadevan, K.; KRishnan, P. (eds.). Methodology for Population Studies and Development. Sage Publications, p. 81-121.

Puyol Antolín, R. (1990). Geografía Humana. Madrid: Pirámide.

RÓdenas Calatayud, C. (1997). "¿Son bajos los flujos migratorios en España?». Revista de Economía Aplicada, 15 (vol. V), p. 155-171.

Rogers, A. (1990). «Requiem for the Net Migrant». Geographical Analysis, 22, p. 283-300. 
Romero González, J.; Albertos Puebla, J.M. (1993). «Retorno al Sur, desconcentración metropolitana y nuevos flujos migratorios en España». Revista Española de Investigaciones Sociológicas, 63, p. 123-144.

Rossi, P.H. (1955). Why Familie. Glencoe, Illinois: The Free Press.

SCHWARTZ, A. (1976). «Migration, Age, and Education». Journal of Political Economy, vol. 84, núm. 4, parte 1, p. 701-719.

SHAW, R.P. (1975). Migration Theory and Fact: Review and Bibliography of Current Literature. Series Bibliográficas, 5. Philadelphia: Regional Science Research Institute.

Shen, J. (1994). «Spatial-Dynamic Population Systems: Analysis and Projection». Environment and Planning $A$, vol. 26, p. 471-488.

Thomlinson, R. (1962). «The Determination of a Base Population for Computing Migration Rates». The Milbank Memorial Fund Quarterly, vol. 40, p. 356-366.

VANDERKAMP, J. (1976). «The Role of Population Size in Migration Studies». Canadian Journal of Economics, vol. 9, núm. 3, p. 508-517.

Vinuesa, J.; Zamora, F.; GénOva, R.; Serrano, P.; Recaño, J. (1994). Demografía. Análisis y Proyecciones. Ed. Síntesis.

Wolpert, J. (1965). "Behavioral Aspects of the Decision to Migrate». Papers and Proceedings of the Regional Science Association, vol. 15, p. 159-169.

ZAX, J.S. (1994). «When is a Move a Migration?». Regional Science and Urban Economics, vol. 24, p. 341-360. 